\title{
ANALISE COMPARATIVA ENTRE DOIS ÂNGULOS CERVICAIS COM A OCLUSÃO EM CRIANÇAS COM E SEM DTM
}

\author{
Comparative analysis between two cervical angles with occlusion \\ in children with and without TMD
}

\author{
Daniela Aparecida Biasotto-Gonzalez ${ }^{(1)}$, Daniela Santos Silva ${ }^{(2)}$, \\ Joelma Magalhães da Costa ${ }^{(3)}$, Cid André Fidelis de Paula Gomes ${ }^{(4)}$, Yasmin El Hage ${ }^{(5)}$, \\ Ana Paula Amaral ${ }^{(6)}$, Fabiano Politti ${ }^{(7)}$,Tabajara de Oliveira Gonzalez ${ }^{(8)}$
}

\section{RESUMO}

Objetivo: comparar dois ângulos cervicais com as classificações oclusais em crianças de 6 a 10 anos com e sem Disfunção Temporomandibular (DTM), identificar se há prevalência de DTM quanto ao gênero, e se há influencia da oclusão sobre os ângulos estudados. Método: realizou-se um estudo observacional transversal com 91 crianças de ambos os gêneros de uma Instituição de Ensino privado, dispostas em Grupo A (sem DTM) e Grupo B (com DTM). Todas as crianças foram avaliadas seguindo uma ficha cadastral constituída de dados referentes a sexo, idade, peso, altura e exame clínico intra-oral para classificação oclusal de Angle. O diagnóstico e a severidade da DTM, foram verificados pelo questionário proposto por Fonseca. Para avaliação dos ângulos cervicais (A1 e A2) foi utilizada a fotogrametria e o Software Alcimagem ${ }^{\circledR}$. Resultados: entre as crianças avaliadas, 52 (57,14\%) apresentaram maloclusão, sendo 29 com DTM e 23 sem DTM, 39 (42,85\%) apresentaram normoclusão, sendo 18 sem DTM e 21 com DTM. Quando comparados os ângulos (A1 e A2) a classe oclusal, foi encontrada diferença significante entre as crianças normoclusivas e maloclusivas com o ângulo A1 $(p=0,04)$. Conclusão: esse estudo demonstrou que o ângulo $A 1$ ( 7 , ATM e Ápice do Mento) foi estatisticamente significante quando associado com a condição oclusal. Não foi encontrado associação, na população estudada, entre ângulo cervical e DTM. Além disso, também foi possível observar que, a prevalência de crianças do gênero feminino com DTM é significantemente maior que a do gênero masculino. Esse estudo demonstrou que a maloclusão pode alterar somente o ângulo A1 e que a DTM não interfere nos ângulos cervicais estudados.

DESCRITORES: Criança; Oclusão Dentária; Postura; Coluna Vertebral; Síndrome da Disfunção da Articulação Temporomandibular

(1) Fisioterapeuta; Docente do programa de mestrado em Ciências da reabilitação - Universidade Nove de Julho UNINOVE, São Paulo, Brasil, Doutora em Biologia e Patologia Buco-Dental pela FOP/UNICAMP.

(2) Graduanda em Fisioterapia, Universidade Nove de Julho - UNINOVE, São Paulo, Brasil.

(3) Fisioterapeuta; Mestre em Ciências da Reabilitação pela Universidade Nove de Julho - UNINOVE, São Paulo, Brasil, Mestre.

(4) Fisioterapeuta; Discente do programa de mestrado em Ciências da Reabilitação, Universidade Nove de Julho UNINOVE, São Paulo, Brasil.

(5) Fisioterapeuta; Discente do programa de mestrado em Ciências da Reabilitação, Universidade Nove de Julho UNINOVE, São Paulo, Brasil.

\section{INTRODUÇÃO}

Como parte do sistema estomatognático, a Articulação Temporomandibular (ATM) tem a função

(6) Fisioterapeuta; Discente do programa de mestrado em Ciências da Reabilitação, Universidade Nove de Julho UNINOVE, São Paulo, Brasil.

(7) Fisioterapeuta; Docente do curso de fisioterapia da Universidade Nove de Julho - UNINOVE, São Paulo, Brasil, Pós-doutor em Morfologia pela UNICAMP.

(8) Fisioterapeuta; Docente do curso de fisioterapia da Universidade Nove de Julho - UNINOVE, São Paulo, Brasil, Doutor em Fisioterapia pela UFSCAR

Conflito de interesses: inexistente 
de possibilitar a fala, a mastigação e a deglutição. Dentre as diversas alterações desse sistema, tem-se a Disfunção Temporomandibular (DTM), termo genérico para um grande número de sinais e sintomas clínicos que acometem o sistema músculo-esquelético na região orofacial principalmente a ATM ${ }^{1,2}$.

Entre os sinais e sintomas mais comuns da DTM encontram-se a dor e/ou desconforto na ATM, nos ouvidos, nos músculos mastigatórios e na cervical de um ou ambos os lados, além de ruídos articulares, amplitude mandibular limitada ou com desvios e dificuldades de mastigação, resultando em complicações estéticas e limitações funcionais dos movimentos mandibulares ${ }^{3,4}$.

$\mathrm{Na}$ população adulta, esses sintomas podem estar presentes em até $37,5 \%$ da população ${ }^{5} \mathrm{com}$ maior prevalência entre 35 a 40 anos $^{6}$. No entanto, alguns estudos epidemiológicos 7,8 , relatam que sinais e sintomas de DTM em crianças e adolescentes são tão comuns quanto em adultos.

Durante a infância, a possibilidade de aparecimento dessa disfunção é elevada no início do desenvolvimento craniofacial e no surgimento de hábitos viciosos e parafuncionais que afetam a oclusão ${ }^{\text {, }}$ músculos mastigatórios, ombros e cervical ${ }^{10,11}$. Dessa maneira, as consequências dessas alterações funcionais nestas estruturas podem ocasionar alterações na posição da cabeça, da mandibula e na oclusão ${ }^{12}$.

Alterações na postura cervical podem alterar as condições oclusais e estas a postura mandibular e a estabilidade da cervical ${ }^{13-15}$. Para comprovar esta relação é necessário a utilização de ferramentas para avaliação postural, como a biofotogrametria computadorizada, que já possui certificação quantitativa ${ }^{16,17}$ para avaliação e confirmação destas relações.

Alguns autores ${ }^{16,18,19}$ têm descrito essa relação entre a postura da cabeça e as alterações do sistema estomatognático. No entanto, esse ainda é um tema controverso e necessita de estudos conduzidos com instrumentos quantitativos e análises precisas em diferentes tipos de populações.

Portanto, a finalidade deste estudo foi comparar dois ângulos cervicais com as classificações oclusais em crianças de 6 a 10 anos com e sem DTM. Identificar se há prevalência de DTM quanto ao gênero, e se há influencia da oclusão sobre os ângulos estudados.

\section{MÉTODO}

Este estudo transversal foi desenvolvido em 91 crianças de ambos os gêneros de uma Instituição de Ensino privada, localizada no interior de São
Paulo. Para o recrutamento e formação de grupos utilizaram-se como critérios de inclusão crianças com faixa etária compreendida entre 6 a 10 anos $(7,73 \pm 1,23)$ cujos pais ou responsáveis legais autorizaram a participação, e crianças com e sem DTM, excluídas as crianças com mordida aberta, mordida cruzada, sobremordida, quadros neurológicos, síndromes, malformações craniofaciais, uso de aparelhos ortodônticos e ortopédicos ou cuja faixa etária não atendesse à proposta da pesquisa e os que se negaram a participar da pesquisa.

Todos os voluntários foram avaliados seguindo uma ficha cadastral constituída de dados referentes a sexo, idade, peso e altura.

Para obtenção do diagnóstico e severidade da DTM, utilizou-se questionário proposto por Fonseca, $1992^{20}$, composto por dez perguntas, com opção de marcar uma alternativa das três respostas contidas no questionário. Soma-se o total de pontos de cada questão, onde: não (0), às vezes (5), sim (10), obtendo-se em seguida o grau de severidade da disfunção, se não há presença de DTM, presença de DTM leve, moderada ou severa.

Nesse estudo, os voluntários foram dispostos em Grupo A (sem DTM) e Grupo B (com DTM). Para a determinação dos indivíduos correspondentes a esses grupos, foi realizado um exame clínico intra-oral por um dentista, conforme a classificação de Angle distribuída por classes I,II e III ${ }^{12}$. Os indivíduos classificados como classe I de Angle foram caracterizados como normoclusivos, e as classes II e III como maloclusivos.

Para o experimento, todos os participantes foram posicionados sempre em uma mesma posição ortostática previamente demarcada para que fosse retiradas fotos nas seguintes posições: vista frontal, posterior, lateral direita e esquerda. Para facilitar a marcação dos ângulos utilizados nesse estudo, os participantes permaneceram com o tronco despido, ou biquíni no caso dos indivíduos do sexo feminino. A câmera fotográfica (Sony ${ }^{\circledR}$ T300 10.1) foi fixada em tripé ajustável a 1,5 metros de distância do participante.

Após a retirada das fotos, foi realizada a medida do ângulo cervical por meio do software Alcimagem ${ }^{\circledR}$ $2.1^{21}$. Os ângulos foram construídos a partir da união entre marcadores semi-esféricos coloridos (1,5 cm de diâmetro), construídos por uma fita dupla face e fixos na pele dos participantes, em pontos pré-estabelecidos sendo esses: C7, ATM, Ápice do Mento. Dessa forma, foi denominado A1 o ângulo formado entre C7, ATM, Ápice do Mento ${ }^{22}$ e A2 o ângulo formado pela interligação entre os marcadores C7, Manúbrio do externo, Ápice do mento ${ }^{23}$ como demonstrado na Figura 1. 


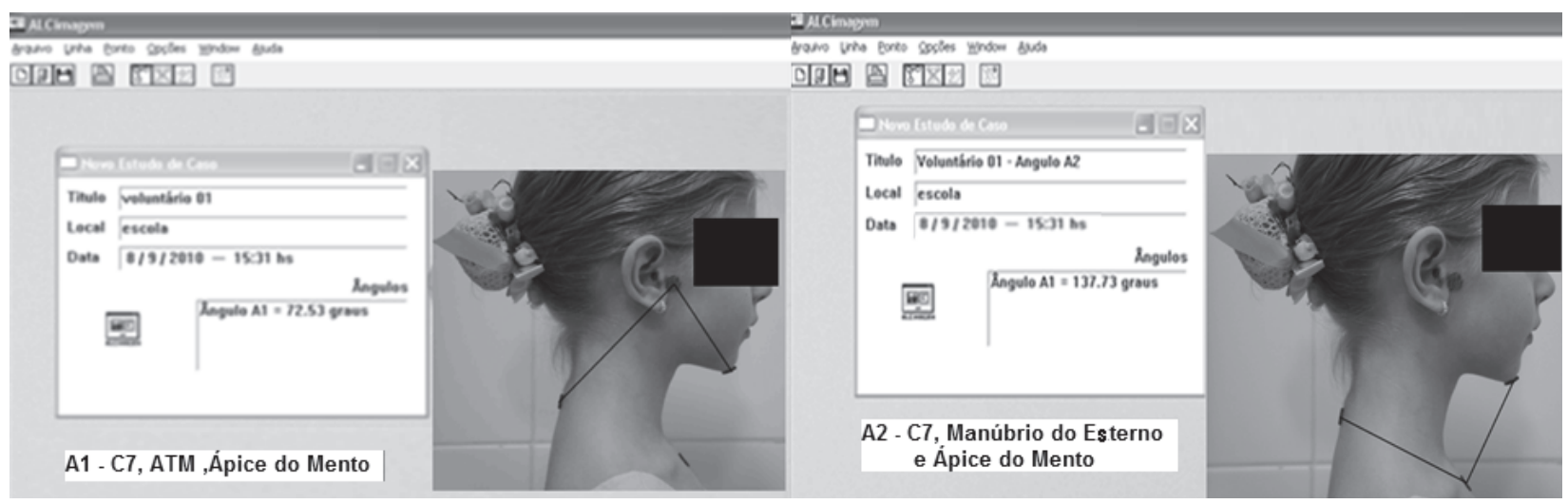

Figura 1 - Ângulo Cervical A1 (C7, ATM, Ápice do Mento) e Ângulo A2 - (C7, Manúbrio do Esterno, Ápice do Mento)

Todas as mensurações dos ângulos foram realizadas pelo mesmo examinador, cego em relação à distribuição dos grupos e os resultados obtidos foram comparados, entre os indivíduos com DTM e sem DTM.

Os pais ou responsáveis dos voluntários receberam todas as informações sobre a pesquisa, assinando uma declaração concordando com a participação dos voluntários na pesquisa, em conformidade com a Resolução 196/96 do Conselho Nacional de Saúde. Este estudo foi aprovado pelo Comitê de Ética em pesquisa Humana da Universidade Nove de Julho sob o n² 245845/2009.

A distribuição dos dados foi verificada pelo teste de Shapiro-Wilk. Dessa forma, para a comparação dos ângulos cervicais (A1 ou A2) entre os indivíduos com normoclusão e maloclusão foi utilizado o teste de Mann Whitney. Para verificar uma possível associação do gênero dos indivíduos com a DTM foi utilizado o teste do $c 2$. Todos os testes estatísticos foram realizados considerando nível de significância $p<0.05$. Todos os dados foram analisados pelo software SPSS 16.0 (SPSS Inc., Chicago, EUA).

\section{RESULTADOS}

A Tabela 1 demonstra a distribuição dos sujeitos por diagnóstico de DTM, idade, peso corporal e altura. $O$ grupo $A$, foi constituído por 41 crianças e o grupo $B$ por 50 , sendo encontrada associação significante do gênero feminino e a presença de DTM (c2 $=4,54$, gl. $1 ; p=0,03$ ) como verificado na Tabela 2.

Tabela 1 - Divisão dos voluntários de acordo com diagnóstico de DTM, sendo Sem DTM Grupo A $(n=41)$, Com DTM Grupo B $(n=50)$

\begin{tabular}{cccc}
\hline Grupo & $\begin{array}{c}\text { Idade } \\
\text { Média (DP) }\end{array}$ & $\begin{array}{c}\text { Peso corporal } \\
\text { Média (DP) }\end{array}$ & $\begin{array}{c}\text { Altura } \\
\text { Média (DP) }\end{array}$ \\
\hline A & $7,75 \pm 1,39$ & $29,55 \pm 7,07$ & $1,31 \pm 0,09$ \\
B & $7,72 \pm 1,09$ & $30,26 \pm 7,77$ & $1,30 \pm 0,07$ \\
\hline
\end{tabular}

DP = Desvio Padrão

Tabela 2 - Distribuição por Gênero nos Grupos A (sem DTM) e B (com DTM).

\begin{tabular}{|c|c|c|c|c|c|}
\hline & & & Grupo A & Grupo B & Total \\
\hline \multirow{4}{*}{ Gênero } & \multirow{2}{*}{ Masculino } & $\mathrm{N}$ & 28 & 23 & 51 \\
\hline & & $\%$ & $30,7 \%$ & $25,3 \%$ & $56,0 \%$ \\
\hline & \multirow{2}{*}{ Feminino } & $\mathrm{N}$ & 13 & 27 & 40 \\
\hline & & $\%$ & $14,3 \%$ & $29,7 \%$ & $44,0 \%$ \\
\hline Total & & & $41(45,0 \%)$ & $50(55,0 \%)$ & $91(100,0 \%)$ \\
\hline
\end{tabular}


A maioria dos avaliados, 52 indivíduos (57,14\%) apresentam Maloclusão, 29 com DTM e 23 sem DTM, 39 indivíduos (42,85\%) apresentaram normoclusão, 18 caracterizados sem DTM e 21 com DTM.

$\mathrm{Na}$ comparação dos ângulos $\mathrm{A} 1$ e $\mathrm{A} 2$ entre os Grupos A e B, não foi encontrada diferença estatisticamente significante (Figura 2).
No entanto, quando comparados os ângulos (A1 e A2) a classe oclusal, foi encontrada diferença estatisticamente significante entre os indivíduos normoclusivos e maloclusivos com o ângulo $A 1(p=0,04)$, apresentando valores médios respectivos, $76,45^{\circ} \mathrm{e}$ $72,78^{\circ}$ (Figura 3).

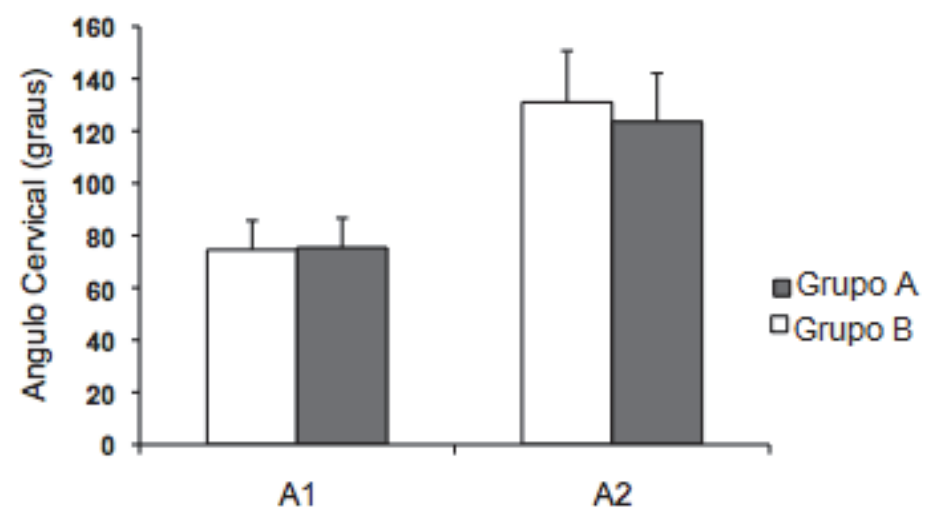

Figura 2 - Média e desvio padrão de dois diferentes ângulos cervicais (A1 e A2) comparados em indivíduos dos Grupos A (Sem DTM) e B (Com DTM)

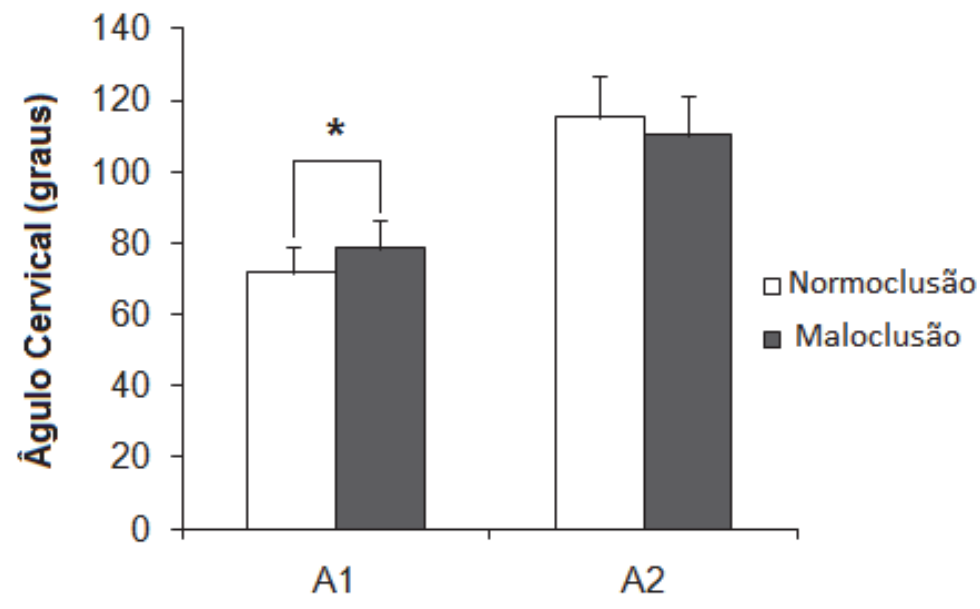

Figura 3 - Média e desvio padrão de dois diferentes ângulos cervicais (A1 e A2) verificados em indivíduos com normoclusão e com maloclusão. *Diferença estatística significante $(p=0,04)$ - Teste de Mann Whitney

\section{DISCUSSÃO}

Neste estudo, a maior parte das crianças que participaram, apresentaram DTM e isso pode estar relacionado com o início do crescimento craniofacial, na faixa etária analisada ${ }^{24}$. Magnusson et al., $2005^{25}$ identificou que alguns fatores oclusais, em especial as classes II e III, são fatores de risco para o desenvolvimento da DTM, resultado este, presente na maioria das crianças avaliadas em seu estudo.

A prevalência de DTM é descrita na literatura, em relação ao gênero, como uma doença que atinge mais as mulheres ${ }^{26}$, e, no presente estudo, dos 91 indivíduos avaliados 40 eram do gênero feminino $(44 \%)$ e 51 eram do gênero masculino 
(56\%), sendo que destes $29,7 \%$ das mulheres apresentaram DTM, sendo estatisticamente mais prevalente, mesmo havendo um número maior de indivíduos do sexo masculino, demonstrando que mesmo sem os fatores de riscos, depressão ${ }^{27}$, estresse ${ }^{28} \mathrm{e}$ em especial para as mulheres, a influência dos hormônios reprodutivos ${ }^{29}$, a população pediátrica do gênero feminino apresentou índices semelhantes aos encontrados na população adulta ${ }^{6}$ além destes resultados concordarem com os resultados descritos na literatura ${ }^{30-32}$ Fato este nos induz observar a necessidade de investigação de outros fatores etiológicos que justifiquem esta tendência presente desde a infância.

A fotogrametria é uma ferramenta importante e eficaz na avaliação da postura da cabeça em pessoas com disfunção temporomandibular ${ }^{10}$, no entanto, no presente estudo não foi encontrada relação significante entre a postura da cabeça e o diagnóstico de DTM, mesmo utilizando dois ângulos cervicais avaliativos distintos.

Em contrapartida, Motta et al., $2009^{33}$, avaliaram e compararam a postura de cabeça e pescoço e a relação com a classe oclusal, entre crianças respiradoras orais e respiradoras nasais e observaram que há relação entre respiração oral, alteração na postura da cabeça e pescoço e oclusão classe II de Angle nas crianças estudadas, sendo a anteriorização da cabeça a alteração mais evidente.

A análise da postura corporal é um aspecto importante como causa ou efeito e pode ser documentada fotograficamente, beneficiando o paciente em seu equilíbrio biopsicossocial. A proposta de documentação com fotos da postura de frente, costas e perfil, junto com análise descritiva e cronológica, é de fundamental importância como material de discussão sob o olhar de cada especialidade, mas de maneira integrada ${ }^{10}$.

Em recente estudo, Motta et al, $2011^{34}$, investigaram a relação entre a postura cervical, por meio da fotogrametria, a presença de bruxismo, em crianças de 3 a 6 anos, e ao analisarem a postura da cabeça, por meio da média do ângulo cervical, os dados apontaram média, estatisticamente significante em relação a um maior ângulo para o grupo de crianças com bruxismo. Dessa maneira, esta informação reafirma as observações na literatura ${ }^{35}$, de que o bruxismo não afeta apenas os músculos mastigatórios, mas também os músculos de todo o complexo craniofacial, ombro e pescoço.

Estudos com crianças ${ }^{36,37}$ têm demonstrado a importância da avaliação oclusal nas diferentes fases dentárias, uma vez que a postura da cabeça pode ser influenciada pela oclusão. Durante a fase de dentição mista, alterações nas características oclusais podem resultar em alterações na postura da cabeça ${ }^{38}$, concordando com os valores obtidos no presente estudo, onde houve diferença entre o ângulo A1 quando comparado crianças com normoclusão com as com maloclusão.

\section{CONCLUSÃO}

Esse estudo demonstrou que o ângulo $A 1(C 7$, ATM e Ápice do Mento) foi estatisticamente significante quando associado com a condição oclusal. Não foi encontrado associação, na população estudada, entre ângulo cervical e DTM. Além disso, também foi possível observar que, a prevalência de crianças do gênero feminino com DTM é significantemente maior que a do gênero masculino. Esse estudo demonstrou que a maloclusão pode alterar somente o ângulo A1 e que a DTM não interfere nos ângulos cervicais estudados.

\section{AGRADECIMENTOS}

Agradecimento especial a Escola PueriDomus por permitir a realização desse estudo. 


\section{ABSTRACT}

Purpose: to compare two cervical angles with occlusal classifications in children between 6 and 10 years old with and without Temporomandibular Disorders (TMD), to identify if there is a prevalence of TMD according to gender, and if there is influence of occlusion on the studied angles. Method: it was conducted a cross sectional observational study with 91 children of both gender in Group A (without TMD) and Group B (TMD). All children were evaluated following a registration form consisted of sex, age, weight, height data and intra-oral clinical examination for occlusal classification of Angle. The diagnosis and severity of TMD were scanned by the questionnaire proposed by Fonseca. For the cervical angles evaluation (A1 and A2) it was used the photogrammetry and the Alcimagem ${ }^{\circledR}$ software. Results: among the evaluated children, $52(57.14 \%)$ presented malocclusion, 29 with TMD and 23 without TMD. 39 (42,85\%) presented normal occlusion, 18 without TMD and 21 with TMD. When the angles were compared, it was found a difference between normal occlusion and malocclusion children with the angle A1 ( $p=0.04)$. Conclusion: this study demonstrated that the angle $A 1(C 7$, TMJ and Mentum Vertex) was statistically significant when associated to the occlusal condition. No association was found in the evaluated population, between TMD and cervical angle. Besides, it was also observed that the prevalence of female children with TMD is significantly larger than the male. This study demonstrated that malocclusion can change only the angle A1 and the TMD does not interfere with the studied cervical angles.

KEYWORDS: Child; Dental Occlusion; Posture; Spine; Temporomandibular Joint Dysfunction Syndrome

\section{REFERÊNCIAS}

1. Mascarenhas $\mathrm{MH}$, Dutra LF. Inter-relações funcionais entre ATM e coluna cervical. In: Maciel RN. ATM e dores craniofaciais - fisiopatologia básica: São Paulo: Santos; 2003.

2. Carrara SV, Conti PCR, Barbosa JS. Termo do 10 Consenso em Disfunção Temporomandibula e Dor Orofacial. Dental Press J Orthod. 2010, 15(3): 114-20.

3. De Boever JA, Nilner M, Orthlieb JD, Steenks MH. Educational Committee of the European Academy of Craniomandibular Disorders. Recommendations by the EACD for examination, diagnosis, and management of patients with temporomandibular disorders and orofacial pain by the general dental practitioner. J Orofac Pain. 2008;22:268-78.

4. Martins RJ, Garcia AR, Garbin CA, Sundefeld MLMM. Associação entre classe econômica e estresse na ocorrência da disfunção temporomandibular. Rev Bras Epidemiol . 2007; 10(2):222.

5. Gonçalves DA, Speciali JG, Jales LC, Camparis $\mathrm{CM}$, Bigal ME. Temporomandibular symptoms, migraine and chronic daily headaches in the population. Neurology. 2009 Aug; 25; 73(8):645-6.

6. Manfredini D; Piccotti F; Ferronato G.; GuardaNardini L. Age peaks of different RDC/TMD diagnoses in a patient population.Journal of Dentistry. 2010; 38(5): 392-9.
7. Thilander BG, Rubio L, Pena C, de Mayorga. Prevalence of temporomandibular dysfunction and its association with malocclusion in children and adolescents: an epidemiologic study related to specified stages of dental development, Angle Orthod. 2002;72(2):146-54.

8. Sonmez H, Sari S, Oksak OG, Camdeviren H, Prevalence of temporomandibular dysfunction in Turkish children with mixed and permanent dentition. J. Oral Rehabil. 2001; 28(3):280-5.

9. Guimarães MMB, Sacco ICN, João SMA. Caracterização postural da jovem praticante de ginástica olímpica. Rev. Brás. Fisioterap. 2007; 11(3): 213-9.

10. Abraao SD, Fornasari CA. Avaliação da posição da cabeça e plano de Frankfut na disfunção da ATM por meio de fotometria. RBO. 2005; 62(1e2): 82-4.

11. Vélez AL, Restrepo CC, Vargas AP, Gallego GJ, Alvarez E, Tamayo V, Tamayo M. Head posture and dental wear evaluation of bruxist children with primary teeth. Journal of Oral Rehabilitation. 2007; 34: 663-70.

12. Faraco CC, Mattos HM. Desvios posturais em indivíduos com maloclusoes Classe I, Classe II e Classe III. Rev. Ter. Man.2008; 23(6): 28-31.

13. Ormeño G, Miralles R, Santander H, Csassus $\mathrm{R}$, Ferrer $\mathrm{P}$, Palazzi C, Moya $\mathrm{H}$. Body position effects on sternocleidomastoid and masseter EMG pattern activity in patients undergoing occlusal splint therapy. Cranio. 1997; 15(4): 300-9. 
14. Goldstein FD, Kraus S, Willian B. Influence of cervical posture on mandibular movement. J Prosthet Dent. 1984; 52(3): 421-6.

15. Milani RS, De Perière DD, Lapeyre L, Pourreyron L. Relationship between dental occlusion and posture. Cranio 2000. 18:127-34.

16. Iunes DH, Castro FA, Salgado AS, Moura IC, Oliveira HS, Bevilaqua GD. Confiabilidade intra e interexaminadores e repetibilidade da avaliação postural pela fotogrametria. Rev Bras Fisioter. 2005;9(3):327-34.

17. Sato TO, Vieira ER, Gil Coury HJC. Análise da confiabilidade de técnicas fotométricas para medir a flexão anterior do tronco. Rev Bras Fisioter. 2003;7(1):53-99.

18. MacConkey $D$. The relationship of posture and dental health. Int J Orofacial Myology. 1991;17:8-10.

19. Solow B, Sonnesen L. Head posture and maloclusions. Eur J Orthod. 1998;20:685-93.

20. Fonseca DM. Disfunção Temporomandibular (DTM): elaboração de um índice anamnésico. Bauru, 1992. Dissertação (Mestrado) Faculdade de Odontologia de Bauru/ USP.

21. Baraúna MA, Ricieri D. Biofotogrametria: recurso diagnóstico do fisioterapeuta. Revista $\mathrm{O}$ Coffito. 2002; 17:7-11.

22. Biasotto-Gonzalez DA. Abordagem interdisciplinardasdisfunçõestemporomandibulares. São Paulo: Manole, 2005.

23. Rocabado M. Biomechanical relationship of the cranial, cervical, and hyoid regions. Journal of Craniomandibular Practice. 1983; 1: 61-6.

24. Santos ECA, Bertoz FA, Pignatta LMB, Arantes FM. Avaliação clínica de sinais e sintomas da disfunção temporomandibular em crianças. Maringá: Rev Dental Press Ortodon Facial. 2006; 11(2): 29-34.

25. Magnusson T, Egernarki I, Carlsson GE. A prospective investigation over two decades on signs and symptoms of temporomandibular disorders and associated variables. A final summary. Acta odontol scand. 2005; 63(2):99-109.

26. Suvinem TI, Reade PC, Kemppainem P, Kononen M, Dworkin SF. Review of aetiological concepts of temporomandibular pain disorders:

http://dx.doi.org/10.1590/s1516-18462012005000067

RECEBIDO EM: 03/05/2011

ACEITO EM: 02/01/2012

Endereço para correspondência:

Daniela Aparecida Biasotto-Gonzalez

Av. Francisco Matarazzo, 612

Água Branca - São Paulo - SP

CEP: 05001-100

E-mail: dani_atm@uninove.br towards a biopsychosocial model for integration of physical disorder factors with psychological illness impact factors. Eur J Pain. 2005; 9(6):613-33.

27. Giannakopoulos NN, Keller L, Rammelsberg P, KronmullerKT,SchimmiterM, Anxietyanddepression in patients with chronic temporomandibular pain and in controls. J Dent. 2010; 38(5): 369-76.

28. McMillian AS, Wong MC, Lee LT, Yeung RW. Depression and diffuse physical symptoms in southern Chinese with temporomandibular disorders. Oral Rehabilitation. 2009; 36(6):403-7.

29. LeResche L. Epidemiology of temporomandibular disorders: implications for the investigation of etiologic factors. Crit Rev Oral Biol Med.1997;8(3):291-305.

30. Monteiro DR, Zuim PR, Pesqueira AA, Ribeiro PD, Garcia AR. Relationship between anxiety and chronic orofacial pain of temporomandibular disorder in a group of university students. J Prosthodont Res. 2010; 55(3):154-8. doi:10.1016/j.jpor.2010.11.001.

31. Egermark-Eriksson, I.; Carlsson, G.E.; Magnusson, T. A 20-year longitudinal study of subjective symptoms of temporomandibular disorders from childhood to adulthood. Acta Odontol Scand. 2001; 59: 40-8.

32. Pedroni CR, DeOliveiraAS, GuaratiniMI. Prevalence study of signs and symptoms of temporomandibular disorders in university students. J oral Rehabi. 2003;30:283-9.

33. Motta LJ, Martins MD, Fernandes KPS, MesquitaFerrari RA, Biasotto-Gonzalez DA,Bussadori SK. Relação da postura cervical e oclusão dentária em crianças respiradoras orais. Rev CEFAC. 2009;11(Supl3):298-304.

34. Motta LJ, Martins MD, Fernandes KPS, MesquitaFerrari RA,Biasotto-Gonzalez DA,Bussadori SK. Craniocervical Posture and Bruxism in Children. Physiother. Res. Int. 2011. Mar;16(1):57-61. doi: 10.1002/pri.478.

35. Velez AL, Restrepo CC, Pelaez-Vargas A,. Gallego GJ, Alvarez E, Tamayo V, Tamayo M. Head posture and dental wear evaluation of bruxist children with primary teeth. Journal of Oral Rehabilitation. 2007 34: 663-70.

36. Gadotti IC, Berzin F, Biasotto-Gonzalez DA. Preliminary rapport on head posture and muscle activity in subjects with class I and II. J Oral Rehabil. 2005;32:794-9.

37. Cesar GM, Tosato J de P, Biasotto-Gonzalez DA. Correlation between occlusion and cervical posture in patients with bruxism. Compend Contin Educ Dent. 2006;27:463-6.

38. Sonnesen Bake M, SolowB. Temporomandibiular disorder in relation to craniofacial dimensiones, head posture and bite force in children select for orthodontic treatment. Eur J Orthod. 2001; 23(2): 179-92. 